\title{
Höchstmengen für Vitamine und Mineralstoffe in Nahrungsergänzungsmitteln
}

\section{Maximum levels for vitamins and minerals in food supplements}

\author{
Anke Weißenborn $^{1}$ (D) Nadiya Bakhiya ${ }^{1} \cdot$ Irmela Demuth $^{1} \cdot$ Anke Ehlers $^{1} \cdot$ \\ Monika Ewald ${ }^{1} \cdot$ Birgit Niemann $^{1} \cdot$ Klaus Richter $^{1} \cdot$ Iris Trefflich $^{1}$ • \\ Rainer Ziegenhagen $^{1} \cdot$ Karen Ildico Hirsch-Ernst $^{1} \cdot$ Alfonso Lampen $^{1}$
}

Received: 29 May 2017/Accepted: 26 October 2017/Published online: 4 January 2018

(c) The Author(s) 2018. This article is an open access publication

Zusammenfassung In Deutschland greift etwa ein Drittel der Erwachsenen regelmäßig zu Nahrungsergänzungsmitteln (NEM). Neben Vitaminen und Mineralstoffen enthalten die Produkte teilweise auch sonstige Stoffe mit physiologischer Wirkung wie Aminosäuren, Fettsäuren, Pflanzenextrakte oder Mikroorganismen. Die Werbung verspricht positive Effekte für Gesundheit, Wohlbefinden und verbesserte Leistungsfähigkeit. Fragen zur Produktsicherheit werden selten thematisiert. Schon im Jahr 2002 wurde in der Europäischen Union (EU) eine Richtlinie (2002/46/EG) zur Angleichung der Rechtsvorschriften der Mitgliedstaaten über NEM erlassen, die auch die Festlegung von Höchstmengen vorsieht. In Deutschland und anderen europäischen Ländern wurden seither verschiedene Modelle für die Höchstmengenableitung für Vitamine und Mineralstoffe entwickelt und diskutiert. Bis heute wurden jedoch auf EU-Ebene keine verbindlichen Höchstmengen festgelegt. Länder wie Dänemark und Frankreich haben daher mittlerweile nationale Regelungen getroffen. Vor diesem Hintergrund hat das Bundesinstitut für Risikobewertung (BfR) auf Basis des derzeitigen wissenschaftlichen Kenntnisstandes Vorschläge für Vitamin- und Mineralstoffhöchstmengen in NEM erarbeitet. Produkte, die diese Empfehlungen einhalten und entsprechend

Anke Weißenborn

Anke.Weissenborn@bfr.bund.de

1 Bundesinstitut für Risikobewertung (BfR), Max-DohrnStr. 8-10, 10589 Berlin, Germany den Herstelleranweisungen eingenommen werden, sind für Personen ab 15 Jahren sicher.

Schlüsselwörter Nahrungsergänzungsmittel · Vitamine · Mineralstoffe · Mikronährstoffe . Höchstdosis · Verbraucherschutz

Abstract About one third of the adults in Germany are using food supplements regularly. Besides vitamins and minerals, these products also contain other substances such as amino acids, fatty acids, plant extracts or microorganisms. Advertising promises positive effects for health, well-being and improved performance. Product safety issues are rarely discussed. Already in the year 2002, a directive $(2002 / 46 / \mathrm{EC})$ on the approximation of the laws of the EU Member States concerning food supplements was adopted, which also provides settings of maximum levels. Since then, different models for the quantitative derivation of maximum levels for vitamins and minerals have been developed and discussed in Germany and other European countries. However, to date no binding maximum levels for vitamins and minerals in food supplements have been set at EU level. Meanwhile, EU member states such as Denmark and France have adopted national regulations. Against this background, the Federal Institute for Risk Assessment (BfR) has developed proposals for maximum levels for vitamins and minerals in food supplements. Vitamin and mineral supplements which comply with these recommendations and are taken according to the manufacturer's instructions are safe for persons from 15 years of age. 
Keywords Food supplement · Vitamins - Minerals . Micronutrients - Maximum daily dose .

Consumer protection

\section{Einleitung}

Deutschland ist mit einem Umsatz von rund 1 Mrd. $€$ von April 2013 bis März 2014 einer der größten Märkte für NEM in der EU, die Tendenz ist steigend. ${ }^{1}$ Etwa jeder dritte Erwachsene nimmt regelmäßig NEM, davon etwa ein Viertel mehr als ein Produkt pro Tag (MRI 2008; Li et al. 2010; Heinemann et al. 2015). NEM werden häufiger von Frauen als von Männern und vor allem von Personen über 35 Jahren mit höherem Bildungsstatus eingenommen. Auch greifen eher Personen mit gesünderem Lebensstil und ausgewogener Ernährung zu NEM (MRI 2008; Li et al. 2010; Rehaag et al. 2013). Neben Vitaminen und Mineralstoffen können MEM auch sonstige Stoffe mit physiologischer Wirkung enthalten. Um die Zusammensetzung und Kennzeichnung der Produkte in der EU zu harmonisieren, war im Juni 2002 vom Europäischen Parlament eine Richtlinie (2002/46/EG) erlassen worden, die 2004 in deutsches Recht überführt wurde (NemV 2004). Demnach sind NEM „Lebensmittel, die dazu bestimmt sind, die normale Ernährung zu ergänzen und die aus Einfach- oder Mehrfachkonzentraten von Nährstoffen oder sonstigen Stoffen mit ernährungsspezifischer oder physiologischer Wirkung bestehen und in dosierter Form in den Verkehr gebracht werden, d. h. in Form von z. B. Kapseln, Pastillen, Tabletten, Pillen und anderen ähnlichen Darreichungsformen, [...] zur Aufnahme in abgemessenen kleinen Mengen“. Geregelt ist auch, welche Vitamine und Mineralstoffe verwendet werden dürfen (Anhang I der Richtlinie) und in welcher chemischen Form (Anhang II der Richtlinie). Schließlich sieht die EU-Richtlinie vor, für Vitamine und Mineralstoffe Höchstmengen festzusetzen, die durch eine Risikobewertung auf Basis allgemein anerkannter wissenschaftlicher Daten und unter Berücksichtigung unterschiedlicher Sensibilitäten einzelner Verbrauchergruppen ermittelt werden sollen. Dies ist bis heute auf EU-Ebene nicht erfolgt.

$\mathrm{Da}$ es sich bei Vitaminen und Mineralstoffen überwiegend um essenzielle Nährstoffe handelt, sind bei der Risikobewertung-im Gegensatz zu

\footnotetext{
$\overline{1}$ https://www.bll.de/de/presse/pressemitteilungen/pm-20141218multivitamine-und-magnesium-die-beliebtesten-nahrungserga enzungsmittel (Zugriff 23.10.2017).
}

Kontaminanten und Rückständen in Lebensmittelnnicht nur Risiken durch eine übermäßige, sondern auch durch eine unzureichende Zufuhr zu berücksichtigen (Bruins et al. 2015). Bei Mikronährstoffen kann daher die klassische toxikologische Vorgehensweise, bei der eine akzeptable tägliche Aufnahmemenge (Acceptable Daily Intake; ADI) auf Basis von Dosis-Wirkungs-Beziehungen und toxikologischen Kenngrößen wie NOAEL (No Observed Adverse Effect Level) und LOAEL (Lowest Observed Adverse Effect Level) und unter Verwendung von Unsicherheitsfaktoren (UF) abgeleitet wird, nicht ohne weiteres angewendet werden. Stattdessen wurde bereits in den 1990er Jahren international das Konzept des sicheren Zufuhrbereichs etabliert (Abb. 1). Dieses sieht vor, dass für jeden Nährstoff ein Zufuhrbereich ermittelt wird, der nach unten durch den jeweiligen Zufuhrreferenzwert bzw. die empfohlene Tageszufuhr (Recommended Daily Allowance; RDA) und nach oben durch die tolerierbare höchste Tagesaufnahmemenge (Tolerable Upper Intake Level, UL) begrenzt wird.

Unter Berücksichtigung dieser Eckpunkte hatte das BfR im Jahr 2004 Höchstmengen für Vitamine und Mineralstoffe in NEM und angereicherten Lebensmitteln abgeleitet (BfR 2004a, b). Auch in anderen europäischen Ländern wurden Modelle zur Höchstmengenableitung entwickelt (Flynn et al. 2003; Rasmussen et al. 2006; Kloosterman et al. 2007; Lyhne Andersen und Tetens 2009; Dufour et al. 2010; Verkaik-Kloosterman et al. 2012). Allen Modellen ist gemein, dass der jeweilige UL als tolerierbare höchste Tagesaufnahmemenge aus allen Quellen berücksichtigt wird und dass die für die zusätzliche Nährstoffaufnahme aus NEM und angereicherten sonstigen Lebensmitteln zur Verfügung stehende sichere Aufnahmemenge durch Bildung der Differenz aus UL und Nährstoffzufuhr aus der üblichen Ernährung (ohne NEM und Anreicherung) abgeleitet wird. Für die Berechnung der sicheren Aufnahmemenge wird eine hohe Zufuhrperzentile (90. oder 95.) herangezogen, um auch Menschen mit hohen Nährstoffaufnahmen aus herkömmlichen Lebensmitteln $\mathrm{zu}$ schützen. Ferner berücksichtigen alle Ansätze die besondere Sensibilität von speziellen Bevölkerungsgruppen. Ungeachtet dieser wissenschaftlichen Vorarbeiten wurde bei den Verhandlungen auf EU-Ebene bislang keine Einigung erzielt, so dass in der EU nach wie vor nicht geregelt ist, in welchen Dosierungen Vitamine und Mineralstoffe in NEM maximal enthalten sein dürfen. Einige Länder wie z.B. Dänemark und Frankreich haben mittlerweile 


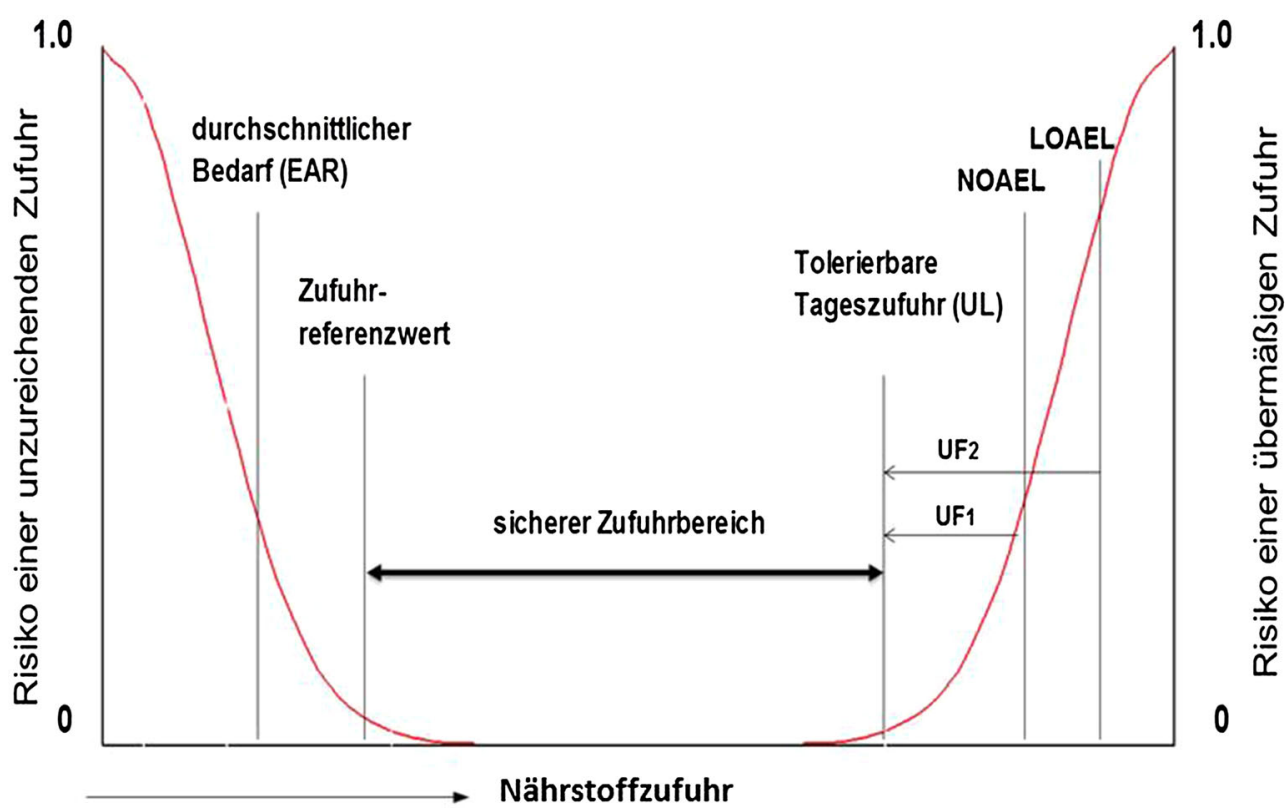

Abb. 1 Sicherer Zufuhrbereich bei Mikronährstoffen (Beziehungen zwischen Zufuhrreferenzwert und LOAEL, NOAEL sowie der Ableitung des UL); modifiziert nach FNB (2001)

nationale Regelungen getroffen. Vor diesem Hintergrund wurden die vom BfR im Jahr 2004 publizierten Höchstmengenempfehlungen (BfR 2004a, b) unter Berücksichtigung neuer wissenschaftlicher Erkenntnisse geprüft und-zunächstfür NEM aktualisiert.

\section{Material und Methoden}

Ausgangspunkt für die Höchstmengenableitung war die Bewertung des Risikos einer unzureichenden oder übermäßigen Mikronährstoffzufuhr in der deutschen Bevölkerung. Entsprechend der gewählten Zielgruppe wurden dafür die in der NVS II mithilfe von Diet History-Interviews erhobenen Nährstoffzufuhrdaten für die Altersgruppe der 14- bis 18-Jährigen sowie für die erwachsene Bevölkerung extrahiert und mit den aktuellen Zufuhrreferenzwerten der D-A-CHGesellschaften (D-A-CH 2015) verglichen. Nur wenn zu einem Nährstoff keine Zufuhrdaten aus der NVS II vorlagen, wurde auf andere publizierte Expositionsdaten zurückgegriffen. Zwar war in der NVS II auch die Einnahme von Supplementen und der Verzehr von angereicherten Lebensmitteln erhoben worden. Da jedoch bei der Auswertung nicht zwischen Konsumenten und Nicht-Konsumenten solcher Produkte differenziert wurde, ist der Einfluss von NEM und angereicherten Lebensmitteln auf die für das NVS II-
Gesamtkollektiv ermittelten Nährstoffzufuhren vernachlässigbar gering.

Zur Abschätzung des gesundheitlichen Risikos einer übermäßigen Zufuhr wurden die von der Europäischen Behörde für Lebensmittelsicherheit (European Food Safety Authority [EFSA] bzw. vom früheren Scientific Committee on Food [SCF]) abgeleiteten ULs für 15- bis 17-Jährige (soweit vorhanden) zugrunde gelegt. Diese Altersgruppe wurde als Bezugsgruppe gewählt, um sicherzustellen, dass die abgeleiteten Höchstmengen nicht nur für Erwachsene sondern auch für Jugendliche, für die häufig niedrigere ULs abgeleitet wurden, sicher sind. Die Berücksichtigung von Kindern unter 15 Jahren hätte aufgrund der noch geringeren ULs keine sinnvollen Höchstmengen ermöglicht.

Durch Bildung der Differenz aus dem UL für 15- bis 17-Jährige und der 95. Zufuhrperzentile (P 95) derselben Altersgruppe wurde für jeden Nährstoff eine Restmenge ( $R_{\text {gesamt }}$ ) abgeleitet, die zusätzlich zur üblichen Ernährung aufgenommen werden kann, ohne dass der jeweilige UL überschritten wird. Bei der Aufteilung der Gesamt-Restmenge auf NEM $\left(R_{\text {NEM }}\right)$ und angereicherte Lebensmittel ( $\left.R_{\text {ang. LM }}\right)$ wurde darauf geachtet, dass die Produkte nicht nur sichere sondern auch signifikante Nährstoffmengen (laut Verordnung (EU) Nr. 1169/2011 mindestens $15 \%$ des jeweiligen Referenzwertes) enthalten. Bei Stoffen mit großen Abständen zwischen dem UL und der 95. 
Zufuhrperzentile wurde die Gesamt-Restmenge zu gleichen Teilen auf NEM und angereicherte Lebensmittel aufgeteilt, während sie bei geringen Abständen (z.B. bei Zink) allein der Kategorie NEM zugeschlagen wurde (Abb. 2). Zur Höchstmengenableitung wurde zusätzlich bei nahezu jedem Nährstoff ein Unsicherheitsfaktor von 2 verwendet, durch den einer möglichen Mehrfachexposition durch die Einnahme unterschiedlicher Produkte Rechnung getragen werden soll (MRI 2008; Li et al. 2010; Heinemann et al. 2015).

Das Verfahren konnte nur angewendet werden, wenn für den betreffenden Nährstoff sowohl ein UL als auch detaillierte Zufuhrdaten verfügbar waren. Bei Nährstoffen, für die diese Daten fehlten, wurden Einzelfallbetrachtungen durchgeführt. Sämtliche Höchstmengenvorschläge beziehen sich auf die vom Hersteller empfohlene Tagesdosis eines NEM.

Die Höchstmengen gelten für Jugendliche ab 15 Jahren und Erwachsene; für Kinder unter 15 Jahren sind gesonderte Betrachtungen und ggf. geringer dosierte Produkte notwendig.

\section{Ergebnisse}

\subsection{Risiko einer unzureichenden Vitamin- oder Mineralstoffzufuhr}

Per definitionem decken die Zufuhrreferenzwerte den Bedarf von nahezu 98 \% einer bestimmten Gruppe der gesunden Bevölkerung. Eine Nährstoffzufuhr in Höhe des Referenzwertes macht eine unzureichende Versorgung sehr unwahrscheinlich. Andererseits ist eine Unterschreitung des Referenzwertes nicht mit einer Unterversorgung gleichzusetzen, sondern erhöht lediglich das Risiko dafür (DGE et al. 2015). Daten aus der NVS II deuten darauf hin, dass Jugendliche ab 15 Jahren und Erwachsene bei den meisten Vitaminen und Mineralstoffen im Median die Zufuhrreferenzwerte erreichen. Ausnahmen sind Folatäquivalente und Vitamin D sowie Jod (unter der theoretischen Annahme, dass in $30 \%$ der verarbeiteten Lebensmittel Jodsalz verwendet wird) sowie bei Frauen zwischen 14 und 18 Jahren Calcium und Eisen (MRI 2008), (Abb. 3 und 4). Auch wenn der Vergleich von Zufuhrmedianen (P 50) mit Zufuhrempfehlungen (P 98) nur eine eingeschränkte Aussagekraft hat, kann die Nährstoffzufuhr auf Bevölkerungsebene näherungsweise immer dann als adäquat angesehen werden, wenn der Zufuhrmedian in Höhe des Referenzwerts liegt.

Für Pantothensäure und Biotin sowie für Fluorid, Mangan, Silizium und Bor konnte das Risiko einer unzureichenden Zufuhr nicht zuverlässig bewertet werden, weil für diese Nährstoffe keine verwertbaren Aufnahmedaten für die deutsche Bevölkerung verfügbar sind und zudem für Silizium und Bor keine Zufuhrreferenzwerte festgelegt wurden.

\subsection{Risiko einer übermäßigen Vitamin- und Mineralstoffzufuhr}

Der UL definiert die tägliche Aufnahmemenge eines Nährstoffs aus allen Quellen, die bei chronischer Zufuhr mit hoher Wahrscheinlichkeit nicht zu negativen gesundheitlichen Wirkungen führt (SCF 2000 zit. in: EFSA 2006). Zwar führt eine Überschreitung des UL nicht zwangsläufig zu unerwünschten Effekten auf die Gesundheit; sie erhöht aber das Risiko dafür.

Betrachtet man für die einzelnen Vitamine und Mineralstoffe die 95. Zufuhrperzentilen und vergleicht diese mit dem jeweiligen UL, so liegen die Ausschöpfungsraten bei Vitamin $\mathrm{D}, \mathrm{E}$ und Niacin (Nicotinsäureamid) bei maximal $15 \%$. Bei Vitamin $B_{6}$, Selen und Molybdän wird der UL durch die normale Ernährung zu ca. $30 \%$ und bei Jod zu 50 bis $60 \%$ ausgeschöpft. Bei Vitamin A, Calcium, Zink und Kupfer liegen dagegen die 95. Zufuhrperzentilen bei 70 bis $100 \%$ des jeweiligen UL (Abb. 5). Für alle anderen Mikronährstoffe konnte das Risiko für eine Überschreitung des UL nicht zuverlässig bewertet werden. Entweder war für diese Stoffe kein UL abgeleitet worden $\left(\beta\right.$-Carotin, Vitamin $\mathrm{K}$, Vitamin $\mathrm{B}_{1}, \mathrm{~B}_{2}$, Pantothensäure, Biotin, Vitamin $\mathrm{B}_{12}$, Vitamin $\mathrm{C}$, Kalium, Eisen, Fluorid und Mangan) oder der UL gilt nur für die zusätzliche Aufnahme über NEM und angereicherte Lebensmittel (Folsäure und Magnesium) und/oder es waren keine Zufuhrdaten für die deutsche Bevölkerung verfügbar (siehe auch Abschnitt 3.1).

Angesichts der in Abb. 5 dargestellten Ausschöpfungsraten muss das Risiko für eine Überschreitung des UL durch supplementierung bei Vitamin A, Calcium, Zink und Kupfer als hoch angesehen werden. Bei Nährstoffen, für die keine numerischen Daten zur Risikoabschätzung vorliegen, kann bei denjenigen, die in bisherigen Untersuchungen am Menschen-selbst bei Aufnahmen weit oberhalb der Zufuhrreferenzwerte-keine nachteiligen Effekte gezeigt haben (z. B. Vitamin $B_{1}, B_{2}$ und $B_{12}$ sowie Biotin und Pantothensäure), nach derzeitigem wissenschaftlichen Kenntnisstand von einem geringen Risiko für unerwünschte Effekte ausgegangen werden. Bei Nährstoffen, die in Humanstudien negative Wirkungen gezeigt haben (selbst wenn keine Dosis-Wirkungsbeziehung abgeleitet werden 


$$
R_{\text {gesamt }}=U L-B A
$$

a) bei Nährstoffen mit Spielraum für die Anreicherung von sonstigen Lebensmitteln

$\rightarrow$ Aufteilung der Gesamt-Restmenge zu gleichen Teilen auf NEM und andere Lebensmittel:

$$
\mathbf{R}_{\text {gesamt }}=\mathbf{R}_{\mathrm{NEM}}+\mathbf{R}_{\text {ang. LM }} \text { bZW. } \quad \mathbf{R}_{\mathrm{NEM}}=\mathbf{R}_{\text {gesamt }} / \mathbf{2}
$$

b) bei Nährstoffen ohne Spielraum für die Anreicherung von sonstigen Lebensmitteln $\rightarrow$ Gesamt-Restmenge wird allein der Kategorie NEM zugeschlagen:

$$
\mathbf{R}_{\text {gesamt }}=\mathbf{R}_{\text {NEM }}
$$

$\rightarrow$ Berücksichtigung eines Unsicherheitsfaktors (UF) von 2 für Mehrfachverwendung von NEM:

$$
\mathrm{THM}_{\text {NEM }}=\mathbf{R}_{\text {NEM }} \text { /UF bZW. THM } \text { NEM }=\mathbf{R}_{\text {NEM }} / 2
$$

Abb. 2 Modell zur Ableitung von Höchstmengen für Mikronährstoffe in Nahrungsergänzungsmitteln (BfR 2004a, b). UL Tolerable Upper Intake Level (Tolerierbare Tageshöchstmenge), in der Regel bezogen auf die tägliche Gesamtnährstoffaufnahme; $B A$ Basisaufnahme über die herkömmliche Ernährung (90. oder 95. Perzentil); $R_{\text {gesamt }}$ Restmenge, die für eine sichere zusätzliche Zufuhr durch NEM und sonstige angereicherte
Lebensmittel insgesamt zur Verfügung steht; $R_{\text {NEM/ang.LM }}$ Sichere Restmenge, die für den Zusatz zu NEM bzw. sonstigen Lebensmitteln zur Verfügung steht; UF Unsicherheitsfaktor bezüglich einer unbekannten, aber möglichen Mehrfacheinnahme von NEM mit gleichen Nährstoffen, $T H M_{\text {NEM }}$ Höchstmenge pro Tagesdosis eines Nahrungsergänzungsmittels

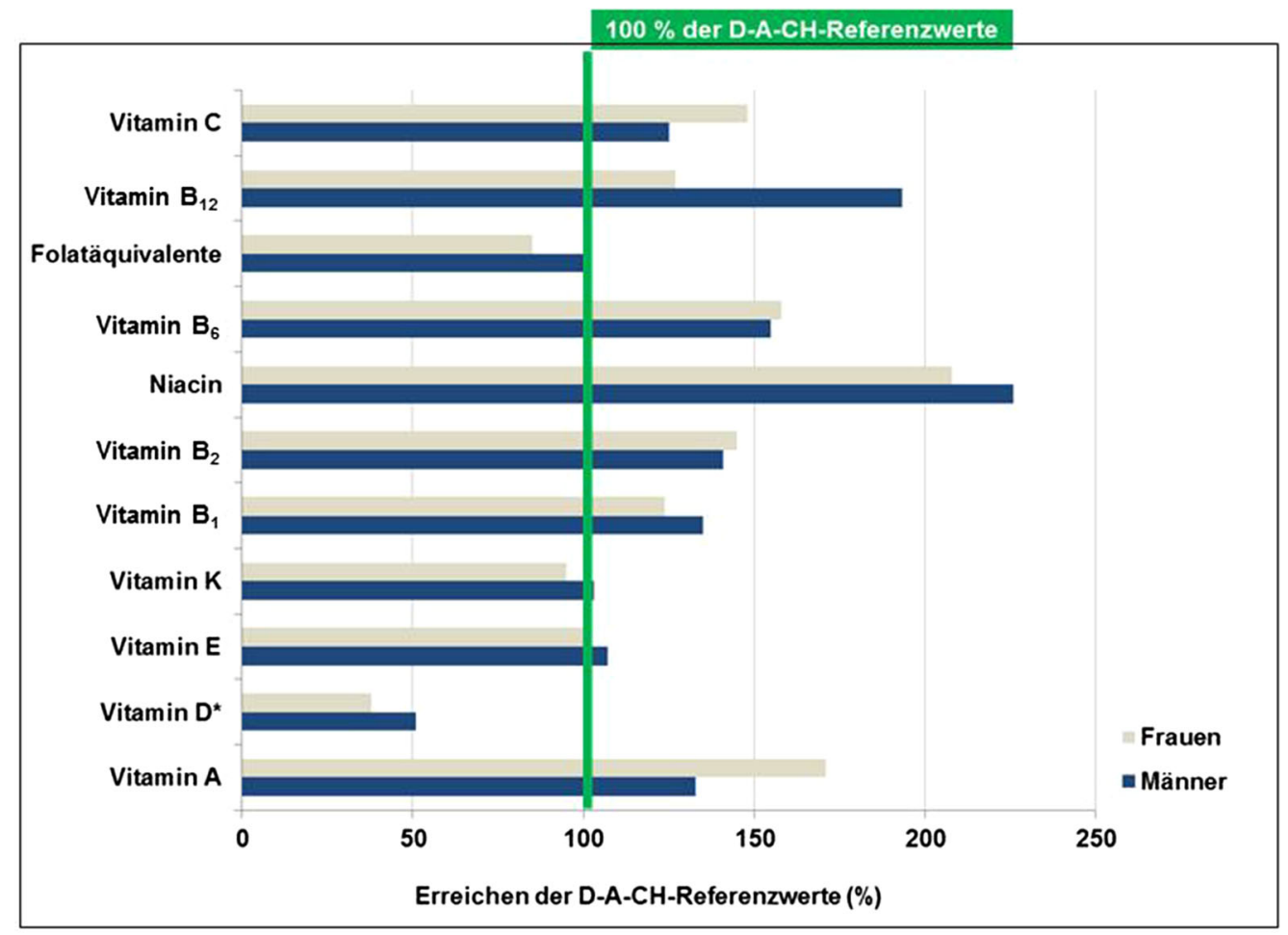

* unter der Annahme, dass $25 \%$ des D.A.CH-Referenzwertes durch die Ernährung erreicht werden können

Abb. 3 Vitaminzufuhr (Mediane) bei Jugendlichen $\geq 15$ Jahren und Erwachsenen über die herkömmliche Ernährung im Vergleich zu den D-A-CH-Referenzwerten (D-A-CH 2015) 


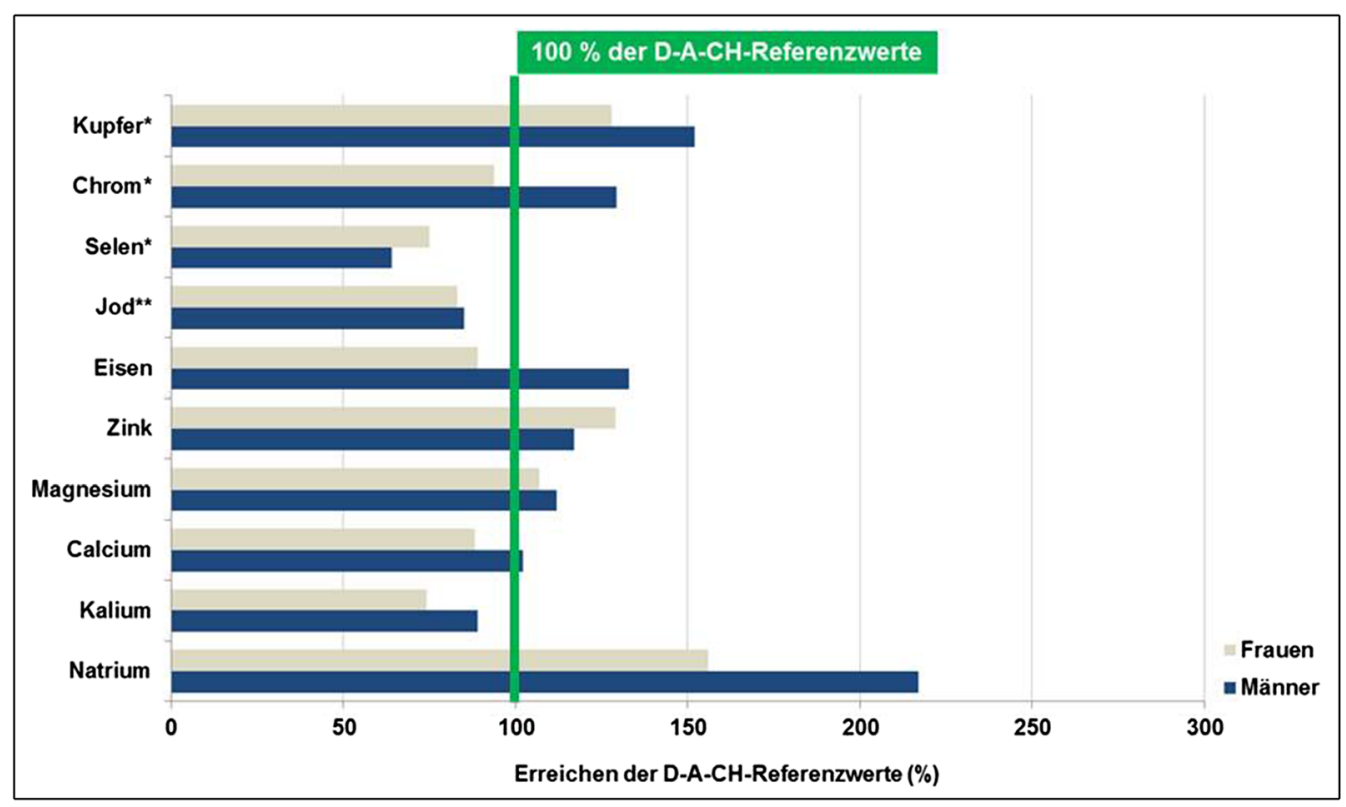

* Nährstoffe, bei denen in Ermanglung von NVS II-Daten auf andere Verzehrerhebungen zurückgegriffen wurde

** Jodzufuhr unter der theoretischen Annahme, dass in $30 \%$ der verarbeiteten Lebensmittel Jodsalz verwendet wird (pers. Mitteilung v. T. Heuer, MRI)

Abb. 4 Mineralstoffzufuhr (Mediane) bei Jugendlichen $\geq 15$ Jahren und Erwachsenen über die herkömmliche Ernährung im Vergleich zu den D-A-CH-Referenzwerten (D-A-CH 2015)

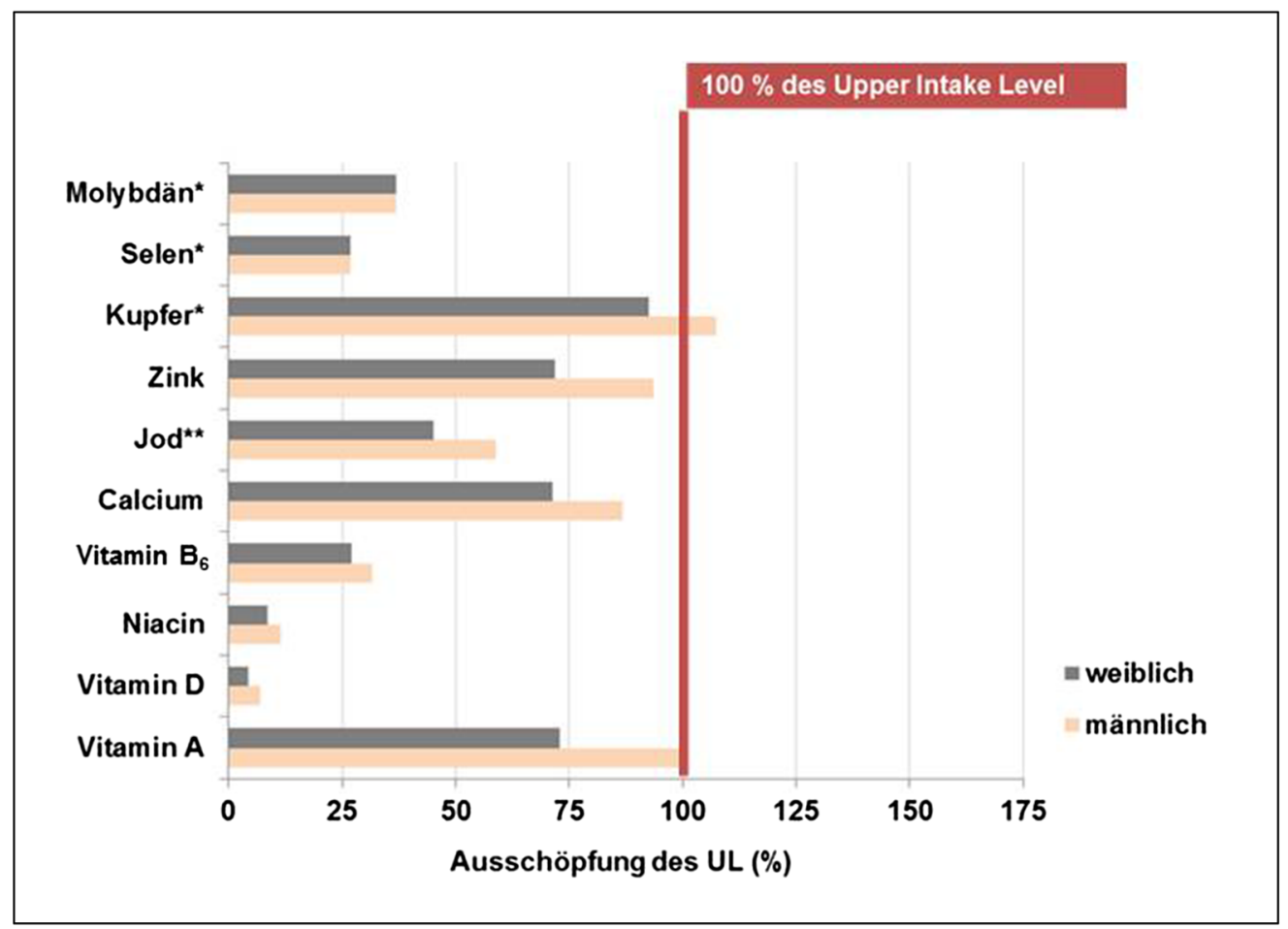

* Nährstoffe, bei denen in Ermanglung von NVS II-Daten auf andere Verzehrerhebungen zurückgegriffen wurde

** Jodzufuhr unter der theoretischen Annahme, dass in $30 \%$ der verarbeiteten Lebensmittel Jodsalz verwendet wird (pers. Mitteilung v. T. Heuer, MRI)

Abb. 5 Ausschöpfung des UL durch die Vitamin- und Mineralstoffzufuhr (P95) über die herkömmliche Ernährung bei Jugendlichen $\geq 15$ Jahren und Erwachsenen 
konnte) oder bei denen Ergebnisse aus Assoziationsstudien und/oder anderen (experimentellen) Studien auf nachteilige Wirkungen hindeuten, besteht dagegen bei Aufnahmen (weit) oberhalb des Zufuhrreferenzwertes ein mäßiges Risiko (z. B. Vitamine $\mathrm{E}, \mathrm{K}$ und $\mathrm{C}$ ) oder ein hohes Risiko (z. B. $\beta$ Carotin, Eisen) für unerwünschte Effekte (siehe auch BfR 2004a, b).

\subsection{Vorschläge für Höchstmengen in NEM}

Das beschriebene Ableitungsverfahren (Abb. 2) war aufgrund von fehlenden Daten oder anderen Überlegungen nur bei einem Teil der Nährstoffe anwendbar: Vitamin D, Niacin, Vitamin $\mathrm{B}_{6}$, Folatäquivalente bzw. Folsäure, Vitamin $B_{12}$, Vitamin C, Kupfer, Kalium, Selen, Chrom, Molybdän, Bor (Tab. 1).

Im Folgenden wird für alle anderen Nährstoffe kurz dargelegt, welche Überlegungen zu den vorgeschlagenen Höchstmengen geführt haben.

\subsubsection{Vitamin A}

Das Ableitungsverfahren ergab eine Restmenge von „Null“, so dass eigentlich kein Spielraum für den Zusatz zu NEM oder sonstigen Lebensmitteln besteht. Vitamin A sollte daher nur von Personen supplementiert werden, die einer zusätzlichen Aufnahme dieses Vitamins tatsächlich bedürfen. Der Höchstwert von $0,4 \mathrm{mg} /$ Tag wurde so gewählt, dass ein NEM einen wirksamen Beitrag zur Vitamin-A-Zufuhr leisten kann, ohne das Risiko für unerwünschte gesundheitliche Effekte in adäquat versorgten Bevölkerungsgruppen erheblich zu erhöhen. Unter Berücksichtigung eines Unsicherheitsfaktors bezüglich möglicher Mehrfachverwendung von Vitamin-A-haltigen NEM entspricht dies einer Höchstmenge von 0,2 mg RE pro Tagesverzehrempfehlung eines NEM. Zusätzlich wird ein Warnhinweis empfohlen, der besagt, dass Vitamin A in der Schwangerschaft nur nach Rücksprache mit dem Arzt eingenommen werden soll.

\subsection{2 $\beta$-Carotin}

Die EFSA hat (2012) eine Stellungnahme zur Verwendung von $\beta$-Carotin als Zusatzstoff abgegeben und kam zu dem Schluss, dass zusätzliche Aufnahmen (als Farbstoff und aus anderen zusätzlichen Quellen) bis zu $15 \mathrm{mg} /$ Tag für die gesamte Bevölkerung, einschließlich Raucher, als sicher angesehen werden können. Dabei wurde geschätzt, dass die
Aufnahme von $\beta$-Carotin als Farbstoff (E 160a) bei durchschnittlich 1-2 mg/Tag liegt (EFSA 2012).

$\beta$-Carotin ist in angereicherten Lebensmitteln verbreitet. Wie Expositionsschätzungen gezeigt haben (Gerber 2014), kann die von der EFSA als sicher bewertete Tagesaufnahmemenge von $15 \mathrm{mg}$ von Hochverzehrern (P95 gemäß NVS II) allein durch angereicherte Fruchtsäfte/-nektare ausgeschöpft werden. Unter Berücksichtigung der derzeitigen Anreicherungspraxis bleibt somit kein Spielraum für den Zusatz von $\beta$-Carotin zu NEM. Nur unter der Bedingung, dass die Anreicherung von herkömmlichen Lebensmitteln mit $\beta$-Carotin künftig so beschränkt wird, dass nur noch ein geringes Risiko für eine Überschreitung des EFSA-Wertes von $15 \mathrm{mg} /$ Tag besteht, könnte ein Zusatz von $\beta$-Carotin zu NEM befürwortet werden.

\subsubsection{Vitamin $E$}

Obwohl für Vitamin E von der EFSA ein UL abgeleitet wurde (260 mg/Tag für 15- bis 17-Jährige) und auch Zufuhrdaten für die deutsche Bevölkerung vorliegen, wurde hier angesichts der kontroversen Datenlage zu negativen gesundheitlichen Effekten durch hohe Vitamin-E-Dosierungen von dem beschriebenen Ableitungsverfahren abgewichen. So ergaben zwei Metaanalysen auf Basis von kontrollierten Interventionsstudien, dass die Supplementierung mit Vitamin E das Risiko für hämorrhagische Schlaganfälle (Vitamin-E-Dosierungen von ca. 130 bis $200 \mathrm{mg} / \mathrm{Tag}$ ) (Schürks et al. 2010) und die Mortalität (Vitamin-EDosierungen im Median: 400 IE bzw. 268 mg/Tag mit einem Risikoanstieg ab 150 IE bzw. $100 \mathrm{mg} / \mathrm{Tag}$ ) (Miller et al. 2005) erhöht hat. Selbst wenn die Datenlage nicht eindeutig ist, wird angesichts der Tatsache, dass mit Vitamin-E-Aufnahmen oberhalb des Bedarfs keine positiven gesundheitlichen Effekte beobachtet wurden, eine Höchstmenge von $30 \mathrm{mg}$ pro Tagesdosis eines NEM (entspricht $200 \%$ des Schätzwertes für eine angemessene Vitamin-E-Zufuhr bei männlichen Personen zwischen 15 und 25 Jahren) als angemessen und sicher angesehen.

\subsubsection{Vitamin $K$}

Aus den in Deutschland vorliegenden Verzehrdaten ergeben sich keine Hinweise auf eine unzureichende Vitamin-K-Aufnahme. Von der EFSA bzw. dem SCF wurde aufgrund von unzureichenden wissenschaftlichen Daten kein UL abgeleitet. Es ist jedoch bekannt, dass bei der Aufnahme von Vitamin K über 
Tabelle 1 Höchstmengenvorschläge für Vitamine und Mineralstoffe, bei denen das vorgeschlagene Ableitungsverfahren angewendet wurde

\begin{tabular}{|c|c|c|c|c|c|c|}
\hline Nährstoff & & $\begin{array}{c}\text { UL } \\
(15-17 \text { J.) }\end{array}$ & $\begin{array}{c}\text { P } 95 \\
\text { (männlich; } \\
14-18 \mathrm{~J} . \text { ) }\end{array}$ & $\begin{array}{l}\text { Ableitungsverfahren } \\
\text { unter Berücksichtigung von Unsicherheitsfakto- } \\
\text { ren (UF) für die Mehrfachexposition }\end{array}$ & $\begin{array}{l}\text { Höchstmen- } \\
\text { genvorschlag }\end{array}$ & $\begin{array}{l}\text { Bemerkungen/ } \\
\text { Empfehlung für } \\
\text { zusätzliche } \\
\text { verpflichtende Angaben }\end{array}$ \\
\hline Vitamin D & $\mu g$ & 100 & 7 & $\begin{array}{l}\qquad 100-7=93 \\
\text { Die Restmenge von } 93 \text { wird zu gleichen Teilen auf } \\
\text { NEM und angereicherte LM aufgeteilt. } \\
\text { Zur Ableitung der NEM-Höchstmenge wurde ein UF } \\
\text { von } 2 \text { verwendet: } \\
\qquad \frac{46,5}{2}=23\end{array}$ & 20 & $\begin{array}{l}\text { Vitamin D-haltige Präpara- } \\
\text { te bis zu einer Tagesdosis } \\
\text { von } 20 \mu g \text { können laut } \\
\text { Expertenkommission } \\
\text { BVL/BfArM noch als NEM } \\
\text { eingestuft werden; Präpa- } \\
\text { rate mit höheren Dosierun- } \\
\text { gen sind als Arzneimittel } \\
\text { anzusehen (BVL/BfArM } \\
\text { 2016) }\end{array}$ \\
\hline Nicotinsäure & $\mathrm{mg}$ & 8 & \multirow{3}{*}{80 (Niacin) } & $\begin{array}{l}\text { Die Niacinaufnahme über die normale Nahrung } \\
\text { kann bei der Höchstmengenableitung unberück- } \\
\text { sichtigt bleiben: } \\
\qquad 8-0=8 \\
\text { Nicotinsäure sollte nicht zur Anreicherung von Le- } \\
\text { bensmitteln verwendet werden. Zur Ableitung der } \\
\text { NEM-Höchstmenge wurde ein UF von } 2 \text { verwendet: } \\
\qquad \frac{8}{2}=4\end{array}$ & 4 & \\
\hline Nicotinamid & $\mathrm{mg}$ & 700 & & $\begin{array}{l}\qquad 700-80=620 \\
\text { Die Restmenge von } 620 \text { wird zu gleichen Teilen auf } \\
\text { NEM und angereicherte LM aufgeteilt. } \\
\text { Zur Ableitung der NEM-Höchstmenge wurde ein UF } \\
\text { von } 2 \text { verwendet: } \\
\qquad \frac{310}{2}=155\end{array}$ & 160 & $\begin{array}{l}\text { Hinweis bei NEM mit mehr } \\
\text { als } 16 \mathrm{mg} / \mathrm{Tag} \text {, dass das } \\
\text { Produkt für Schwangere } \\
\text { nicht geeignet ist }\end{array}$ \\
\hline Inosithexanicotinat & $\mathrm{mg}$ & - & & $\begin{array}{l}\text { Angesichts der Bewertung von Inosithexanicotinat } \\
\text { durch die EFSA (2009a) wird hier analog zum Vor- } \\
\text { gehen bei Nicotinsäure verfahren }\end{array}$ & 4 & \\
\hline Vitamin $B_{6}$ & $\mathrm{mg}$ & 20 & 6,3 & $\begin{array}{l}\qquad 20-6,3=13,7 \\
\text { Die Restmenge von } 13,7 \text { bzw. } 14 \text { wird zu gleichen } \\
\text { Teilen auf NEM und angereicherte LM aufgeteilt. } \\
\text { Zur Ableitung der NEM-Höchstmenge wurde ein UF } \\
\text { von } 2 \text { verwendet: } \\
\qquad \frac{7}{2}=3,5\end{array}$ & 3,5 & \\
\hline Folsäure & $\mu g$ & 800 & - & $\begin{array}{l}\text { Da der UL nur für synthetische Folsäure gilt, kann } \\
\text { die Aufnahme von Folat über die normale Nahrung } \\
\text { außer Acht gelassen werden: } \\
\qquad 800-0=800 \\
\text { Die Restmenge von } 800 \text { wird zu gleichen Teilen auf } \\
\text { NEM und angereicherte LM aufgeteilt. Zur Ableitung } \\
\text { der NEM-Höchstmenge wurde ein UF von } 2 \text { ver- } \\
\text { wendet: } \\
\qquad \frac{400}{2}=200\end{array}$ & 200 & $\begin{array}{l}\text { Für Frauen mit Kinder- } \\
\text { wunsch und im ersten } \\
\text { Drittel der Schwanger- } \\
\text { schaft: } 400 \mu \mathrm{g} / \mathrm{Tag}\end{array}$ \\
\hline Vitamin $B_{12}$ & $\mu g$ & $\begin{array}{l}\text { laut SCF (2000) sind } \\
\text { bei zusätzlicher Auf- } \\
\text { nahme bis zu } \\
100 \mu g / T a g \text { keine } \\
\text { unerwünschten } \\
\text { Wirkungen bekannt } \\
\text { geworden }\end{array}$ & & $\begin{array}{l}\text { Die Aufnahme von Vitamin B12 über die normale } \\
\text { Nahrung kann bei der Höchstmengenableitung } \\
\text { unberücksichtigt bleiben: } \\
\qquad 100-0=100 \\
\text { Die Restmenge von } 100 \text { wird zu gleichen Teilen auf } \\
\text { NEM und angereicherte LM aufgeteilt. Zur Ableitung } \\
\text { der NEM-Höchstmenge wurde ein UF von } 2 \text { ver- } \\
\text { wendet: } \\
\qquad \frac{50}{2}=25\end{array}$ & 25 & \\
\hline Vitamin C & $\mathrm{mg}$ & $\begin{array}{l}- \\
\text { bei einer zusätzlichen } \\
\text { Aufnahme von } \\
\mathbf{1 0 0 0} \mathbf{m g} / \mathrm{Tag} \text { (Orien- } \\
\text { tierungswert) sind laut }\end{array}$ & - & $\begin{array}{l}\text { Die Vitamin-C-Aufnahme über die normale Nahrung } \\
\text { kann bei der Höchstmengenableitung unberücksich- } \\
\text { tigt bleiben: } \\
\qquad 1000-0=1000\end{array}$ & 250 & \\
\hline
\end{tabular}


Tabelle 1 continued

\begin{tabular}{|c|c|c|c|c|c|c|}
\hline Nährstoff & & $\begin{array}{c}\text { UL } \\
(15-17 \text { J.) }\end{array}$ & $\begin{array}{c}\text { P } 95 \\
\text { (männlich; } \\
14-18 \mathrm{~J} . \text { ) }\end{array}$ & $\begin{array}{l}\text { Ableitungsverfahren } \\
\text { unter Berücksichtigung von Unsicherheitsfakto- } \\
\text { ren (UF) für die Mehrfachexposition }\end{array}$ & $\begin{array}{l}\text { Höchstmen- } \\
\text { genvorschlag }\end{array}$ & $\begin{array}{l}\text { Bemerkungen/ } \\
\text { Empfehlung für } \\
\text { zusätzliche } \\
\text { verpflichtende Angaben }\end{array}$ \\
\hline & & $\begin{array}{l}\text { EFSA keine adversen } \\
\text { (gastrointestinalen) } \\
\text { Effekte zu erwarten }\end{array}$ & & $\begin{array}{l}\text { Die Restmenge von } 1000 \text { wird zu gleichen Teilen } \\
\text { auf NEM und angereicherte LM aufgeteilt. Zur Ablei- } \\
\text { tung der NEM-Höchstmenge wurde ein UF von } 2 \\
\text { verwendet: } \\
\qquad \frac{500}{2}=250\end{array}$ & & \\
\hline Eisen & $\mathrm{mg}$ & $\begin{array}{l}\text { für Frauen zwischen } \\
14 \text { und } 50 \text { Jahren } \\
\text { wurde behelfsmäßig } \\
\text { der UL des ehemali- } \\
\text { gen US- } \\
\text { amerikanischen Insti- } \\
\text { tute of Medicine } \\
\text { (IOM) von } \mathbf{4 5} \mathbf{~ m g / T a g} \\
\text { herangezogen }\end{array}$ & $20,6^{\text {a) }}$ & $\begin{array}{l}\qquad 45-21=24 \\
\text { Die Restmenge von } 25 \text { wird zu gleichen Teilen auf } \\
\text { NEM und angereicherte LM aufgeteilt. Zur Ablei- } \\
\text { tung der NEM-Höchstmenge wurde ein UF von } 2 \\
\text { verwendet: } \\
\qquad \frac{12}{2}=6\end{array}$ & 6 & $\begin{array}{l}\text { Warnhinweis auf eisenhal- } \\
\text { tigen NEM, dass Männer, } \\
\text { postmenopausale Frauen } \\
\text { und Schwangere Eisen nur } \\
\text { nach Rücksprache mit } \\
\text { ihrem Arzt einnehmen } \\
\text { sollten }\end{array}$ \\
\hline Kupfer & $\mathrm{mg}$ & $\begin{array}{c}4(\geq 15-18 \mathrm{~J} .) \\
5(>18 \mathrm{~J} .)\end{array}$ & $\begin{array}{l}4,3^{\text {b) }} \\
2,8^{c)}\end{array}$ & $\begin{array}{l}\qquad 4-4,3=-0,3 \\
\qquad 5-2,8=2,2 \\
\text { Die geringe Restmenge von 2,2 (für Personen > } 18 \\
\text { J.) sollte nur für NEM verwendet werden. Zur Ablei- } \\
\text { tung der Höchstmenge wurde ein UF von } 2 \text { verwen- } \\
\text { det: } \\
\qquad \frac{2,2}{2}=1,1\end{array}$ & $\begin{array}{l}0(15-17 \mathrm{~J} .) \\
1(>18 \mathrm{~J} .)\end{array}$ & $\begin{array}{l}\text { Warnhinweis: } \\
\text { Für Kinder und Jugendli- } \\
\text { che nicht geeignet }\end{array}$ \\
\hline Kalium & $\mathrm{mg}$ & $\begin{array}{l}\text { das BfR hat im Jahr } \\
2004 \text { einen Orientie- } \\
\text { rungswert von } \\
1000 \mathrm{mg} / \mathrm{Tag} \text { für die } \\
\text { zusätzliche Zufuhr } \\
\text { abgeleitet }\end{array}$ & - & $\begin{array}{l}\text { Die Kaliumaufnahme über die normale Nahrung } \\
\text { kann bei der Höchstmengenableitung unberücksich- } \\
\text { tigt bleiben: } \\
\qquad 1000-0=1000 \\
\text { Die Restmenge von } 1000 \text { sollte nur für NEM ver- } \\
\text { wendet werden. Zur Ableitung der Höchstmenge } \\
\text { wurde ein UF von } 2 \text { verwendet: } \\
\qquad \frac{1000}{2}=500\end{array}$ & 500 & \\
\hline Selen & $\mu \mathrm{g}$ & 250 & $67,4^{\text {d) }}$ & $\begin{array}{l}\qquad 250-67,4=182,6 \\
\text { Die Restmenge von } 180 \text { wird zu gleichen Teilen auf } \\
\text { NEM und angereicherte LM aufgeteilt. Zur Ableitung } \\
\text { der NEM-Höchstmenge wurde ein UF von } 2 \text { ver- } \\
\text { wendet: } \\
\qquad \frac{90}{2}=45\end{array}$ & 45 & \\
\hline Chrom & $\mu \mathrm{g}$ & $\begin{array}{l}\text { bei einer zusätzlichen } \\
\text { Aufnahme von } 250 \\
\mu \text { g/Tag (Orientie- } \\
\text { rungswert) sind laut } \\
\text { EFSA keine adversen } \\
\text { Effekte zu erwarten }\end{array}$ & - & $\begin{array}{l}\text { Die Aufnahme von Chrom über die normale Ernäh- } \\
\text { rung kann bei der Höchstmengenableitung vernach- } \\
\text { lässigt werden: } \\
\qquad 250-0=250 \\
\text { Die Restmenge von } 250 \text { wird zu gleichen Teilen auf } \\
\text { NEM und angereicherte LM aufgeteilt. Zur Ableitung } \\
\text { der NEM-Höchstmenge wurde ein UF von } 2 \text { ver- } \\
\text { wendet: } \\
\qquad \frac{125}{2}=62,5\end{array}$ & 60 & \\
\hline Molybdän & $\mu \mathrm{g}$ & 500 & $\left.185,5^{\mathrm{e}}\right)$ & $\begin{array}{l}\qquad 500-185,5=314,5 \\
\text { Die Restmenge von } 314 \text { wird zu gleichen Teilen auf } \\
\text { NEM und angereicherte LM aufgeteilt. Zur Ableitung } \\
\text { der NEM-Höchstmenge wurde ein UF von } 2 \text { ver- } \\
\text { wendet: } \\
\qquad \frac{157}{2}=78,5\end{array}$ & 80 & \\
\hline Bor & $\mathrm{mg}$ & $\begin{array}{l}9(15-17 \mathrm{~J} .) \\
10(\geq 18 \mathrm{~J} .)\end{array}$ & $9^{f)}$ & $\begin{array}{l}\qquad 9-9=0 \\
\qquad 10-9=1 \\
\text { Die geringe Restmenge von } 1 \text { (für Personen > 18 J.) } \\
\text { sollte nur für NEM verwendet werden. Zur Ableitung } \\
\text { der NEM-Höchstmenge wurde ein UF von } 2 \text { ver- } \\
\text { wendet: } \\
\qquad \frac{1}{2}=0,5\end{array}$ & $\begin{array}{l}0(15-17 \mathrm{~J} .) \\
0,5(>18 \mathrm{~J} .)\end{array}$ & $\begin{array}{l}\text { Warnhinweis: } \\
\text { Für Kinder und Jugendli- } \\
\text { che nicht geeignet }\end{array}$ \\
\hline
\end{tabular}

a hier: Frauen zwischen 14 und 18 Jahren

b Aufnahmedaten für 12- bis 17-Jährige aus der Ernährungsstudie als KiGGS-Modul (EsKiMo) (Mensink et al. 2007)

c Daten aus einer Expositionsschätzung der EFSA (2015) zur Kupferzufuhr in Europa

d Daten aus einer Expositionsschätzung der EFSA (2014b) zur Selenzufuhr bei 10- bis < 18-Jährigen in Europa

e Daten aus einer Expositionsschätzung der EFSA (2013) zur Molybdänzufuhr in Europa

${ }^{f}$ Daten aus einer Expositionsschätzung des BfR (2006) zu Boraufnahmen der deutschen Bevölkerung 
Tabelle 2 Zufuhrreferenzwerte und Tolerable Upper Intake Levels (UL) für Vitamine im Vergleich zu Aufnahmemengen über die herkömmliche Nahrung sowie Höchstmengenvorschläge für Vitamine, bei denen das Ableitungsverfahren nicht anwendbar war

\begin{tabular}{|c|c|c|c|c|c|c|c|}
\hline \multirow{2}{*}{ Nährstoff } & & \multirow{2}{*}{$\begin{array}{c}\text { D-A-CH- } \\
\begin{array}{c}\text { Zufuhrreferenz } \\
\text { wert }\end{array} \\
(15-\geq 65 \text { J.) } \\
\end{array}$} & \multirow{2}{*}{ 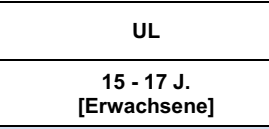 } & \multicolumn{2}{|c|}{$\begin{array}{c}\text { Nährstoffaufnahme } \\
\text { über normale Nahrung (14-80 J.) }\end{array}$} & \multirow{2}{*}{$\begin{array}{l}\text { Höchstmengenvor } \\
\text { schlag }\end{array}$} & \multirow{2}{*}{$\begin{array}{l}\text { Bemerkungen/ } \\
\text { Empfehlung für zusätzliche } \\
\text { verpflichtende Angaben }\end{array}$} \\
\hline & & & & P50 & P95 & & \\
\hline Vitamin A & $\mathrm{mg}$ & $0,8-1,1$ & $2,6[3,0]$ & $\begin{array}{l}0,6-0,7(\mathrm{~m}) \\
0,4-0,5(\mathrm{w})\end{array}$ & $\begin{array}{l}2,6-2,9(m) \\
1,7-2,0(w)\end{array}$ & 0,2 & $\begin{array}{l}\text { Warnhinweis, der besagt, dass Vitamin } \\
\text { A in der Schwangerschaft nur nach } \\
\text { Rücksprache mit dem Arzt } \\
\text { eingenommen werden soll } \\
\text { Kein Spielraum für Anreicherung von } \\
\text { sonstigen Lebensmitteln }\end{array}$ \\
\hline$\beta$-Carotin & $\mathrm{mg}$ & $2-4$ & $\begin{array}{l}\text { laut EFSA }(2012) \text { ist die } \\
\text { zusätzliche Aufnahme von } \\
15 \mathrm{mg} / \mathrm{Tag}, \mathrm{z.B} . \text { über NEM } \\
\text { und Farbstoffe, sicher }\end{array}$ & $\begin{array}{l}3,8-4,4(m) \\
3,9-4,7(w)\end{array}$ & $\begin{array}{l}10,0-13,0(m) \\
11,1-13,4(w)\end{array}$ & \multicolumn{2}{|c|}{$\begin{array}{l}\text { Zusatz von } \beta \text {-Carotin zu NEM nur unter der Bedingung, dass } \\
\text { auf die Anreicherung alkoholfreier Getränke verzichtet oder } \\
\text { diese beschränkt wird }\end{array}$} \\
\hline Vitamin E & $\mathrm{mg}$ & $11-15$ & 260 [300] & $\begin{array}{l}12,4-16,2(m) \\
11,3-13,0(w)\end{array}$ & $\begin{array}{l}24,6-39,3(m) \\
23,0-35,8(w)\end{array}$ & 30 & \\
\hline Vitamin K & $\mu g$ & $65-80$ & -- & $\begin{array}{l}63-85(m)^{a)} \\
56-79(w)^{a)}\end{array}$ & -- & 80 & $\begin{array}{l}\text { Personen, die gerinnungshemmende } \\
\text { Arzneimittel (vom Cumarin-Typ) } \\
\text { einnehmen, sollten vor dem Verzehr } \\
\text { von Vitamin-K-haltigen NEM ihren Arzt } \\
\text { befragen }\end{array}$ \\
\hline Vitamin $B_{1}$ & $\mathrm{mg}$ & $1,0-1,4$ & - & $\begin{array}{l}1,3-1,9(m) \\
0,9-1,0(w)\end{array}$ & $\begin{array}{l}2,4-4,9(m) \\
2,0-3,2(w)\end{array}$ & - & $\begin{array}{l}\text { vorläufig kann auf die Festlegung einer } \\
\text { Höchstmenge verzichtet werden }\end{array}$ \\
\hline Vitamin $B_{2}$ & $\mathrm{mg}$ & $1,0-1,6$ & -- & $\begin{array}{l}1,6-2,2(m) \\
1,4-1,7(w)\end{array}$ & $\begin{array}{l}3,0-6,0(m) \\
2,7-4,5(w)\end{array}$ & -- & $\begin{array}{l}\text { vorläufig kann auf die Festlegung einer } \\
\text { Höchstmenge verzichtet werden }\end{array}$ \\
\hline $\begin{array}{l}\text { Pantothen- } \\
\text { säure }\end{array}$ & $\mathrm{mg}$ & 6 & - & - & - & - & $\begin{array}{l}\text { vorläufig kann auf die Festlegung einer } \\
\text { Höchstmenge verzichtet werden }\end{array}$ \\
\hline Biotin & $\mu \mathrm{g}$ & $30-60$ & -- & -- & -- & - & $\begin{array}{l}\text { vorläufig kann auf die Festlegung einer } \\
\text { Höchstmenge verzichtet werden }\end{array}$ \\
\hline
\end{tabular}

a Daten aus dem Ernährungsbericht 2012, basierend auf 24-h-Recalls in der NVS II (DGE 2012)

NEM Interaktionen mit gerinnungshemmenden Arzneimitteln vom Cumarintyp möglich sind, die für die Betroffenen mit einer Abschwächung des therapeutischen Effektes der Arzneimittel verbunden sein und dadurch zu unerwünschten gesundheitlichen Wirkungen führen können. Da im Zusammenhang mit der im Jahr 2004 vom BfR (2004a) vorgeschlagenen Höchstmenge von $80 \mu \mathrm{g} / \mathrm{Tag}$ bis heute keine unerwünschten Effekte bekannt geworden sind und keine neuen wissenschaftlichen Erkenntnisse vorliegen, wird dieser Wert als vorläufig tolerabel angesehen. Jedoch wird für NEM mit Vitamin $K$ zusätzlich ein Warnhinweis empfohlen: Personen, die gerinnungshemmende Arzneimittel (vom Cumarintyp) einnehmen, sollen demnach vor dem Verzehr von Vitamin-K-haltigen NEM ihren Arzt befragen.

\subsubsection{Vitamine $B_{1}, B_{2}$, Biotin und Pantothensäure}

Für die Vitamine $B_{1}, B_{2}$, Biotin und Pantothensäure wurden keine ULs abgeleitet (EFSA 2006). Bislang wurden für diese Vitamine auch in Mengen, die weit oberhalb der Zufuhrreferenzwerte liegen, keine nachteiligen gesundheitlichen Effekte beobachtet. Selbst wenn daraus nicht geschlussfolgert werden kann, dass diese Stoffe kein Potenzial für unerwünschte Effekte haben, kann auf der Basis des derzeitigen Kenntnisstandes auf die Festlegung von Höchstmengen für diese Vitamine verzichtet werden.

\subsubsection{Natrium, Chlorid, Phosphor}

Es sind keine Gründe erkennbar, die für eine Verwendung von Natrium, Chlorid und Phosphor in NEM zu ernährungsphysiologischen Zwecken sprechen. Denkbar ist jedoch ein indirekter Zusatz durch die Verwendung von Vitamin- oder anderen Mineralstoffverbindungen, die diese drei Mineralstoffe als Begleitionen enthalten können (siehe Anhang II der Richtlinie 2002/46/EG). In diesen Fällen ergibt sich eine Mengenbeschränkung für Natrium, Chlorid und Phosphor aus den für die primären Mikronährstoffzusätze festgelegten Höchstmengen.

\subsubsection{Calcium und Zink}

Bei Anwendung des Ableitungsverfahrens ergeben sich für Calcium und Zink Restmengen, die keine 
Tabelle 3 Zufuhrreferenzwerte und Tolerable Upper Intake Levels (UL) für Mineralstoffe im Vergleich zu Aufnahmemengen über die herkömmliche Nahrung sowie Höchstmengenvorschläge für Mineralstoffe, bei denen das Ableitungsverfahren nicht anwendbar war

\begin{tabular}{|c|c|c|c|c|c|c|c|}
\hline \multirow[t]{2}{*}{ Nährstoff } & & \multirow{2}{*}{$\begin{array}{l}\text { D-A-CH- } \\
\text { Zufuhr- } \\
\text { referenz- } \\
\text { wert } \\
(15-\geq 65 \mathrm{~J} .)\end{array}$} & \multirow{2}{*}{$\begin{array}{c}\text { UL } \\
\ldots \ldots \ldots \ldots \ldots \\
15-17 \mathrm{~J} . \\
{[\text { Erwachsene] }}\end{array}$} & \multicolumn{2}{|c|}{$\begin{array}{c}\text { Nährstoffaufnahme } \\
\text { über normale Nahrung } \\
(14-80 \mathrm{~J} .)\end{array}$} & \multirow{2}{*}{$\begin{array}{l}\text { Höchstmengen- } \\
\text { vorschlag }\end{array}$} & \multirow{2}{*}{$\begin{array}{l}\text { Bemerkungen/ } \\
\text { Empfehlung für zusätzliche } \\
\text { verpflichtende Angaben }\end{array}$} \\
\hline & & & & P50 & P95 & & \\
\hline Natrium & g & 1,5 & - & $\begin{array}{l}2,9-3,4(m) \\
2,2-2,4(w)\end{array}$ & $\begin{array}{l}4,8-6,7(m) \\
3,6-4,4(w)\end{array}$ & 0 & $\begin{array}{l}\text { Kein Zusatz zu ernährungs- } \\
\text { physiologischen Zwecken }\end{array}$ \\
\hline Chlorid & $g$ & 2,3 & -- & -- & -- & 0 & $\begin{array}{l}\text { Kein Zusatz zu ernährungs- } \\
\text { physiologischen Zwecken }\end{array}$ \\
\hline Calcium & g & $1,0-1,2$ & $\begin{array}{l}2,5 \\
\text { (UL wurde nur für } \\
\text { Erwachsene } \\
\text { abgeleitet) }\end{array}$ & $\begin{array}{l}0,9-1,2(m) \\
0,9-1,0(w)\end{array}$ & $\begin{array}{l}1,7-2,4(m) \\
1,5-1,8(w)\end{array}$ & 0,5 & $\begin{array}{l}\text { Hinweis bei NEM mit mehr als } \\
250 \mathrm{mg} \text { Calcium/Tag, dass auf } \\
\text { die Einnahme weiterer } \\
\text { calciumhaltiger NEM verzichtet } \\
\text { werden soll }\end{array}$ \\
\hline Phosphor & $\mathrm{mg}$ & $700-1250$ & -- & -- & -- & 0 & $\begin{array}{l}\text { Kein Zusatz zu ernährungs- } \\
\text { physiologischen Zwecken }\end{array}$ \\
\hline Magnesium & $\mathrm{mg}$ & $300-400$ & $\begin{array}{c}250 \\
\text { (UL gilt nur für } \\
\text { zusätzliche } \\
\text { Zufuhr) }\end{array}$ & $\begin{array}{l}389-454 \\
334-378\end{array}$ & $\begin{array}{l}596-809(m) \\
537-594(w)\end{array}$ & 250 & $\begin{array}{l}\text { Die Aufnahme soll auf zwei oder } \\
\text { mehr Portionen pro Tag verteilt } \\
\text { werden }\end{array}$ \\
\hline Eisen & $\mathrm{mg}$ & $10-15$ & -- & $\begin{array}{l}13,1-15,1(m) \\
11,0-12,3(w)\end{array}$ & $\begin{array}{l}21,2-28,3(m) \\
17,6-20,6(w)\end{array}$ & 6 & $\begin{array}{l}\text { Warnhinweis auf eisenhaltigen } \\
\text { NEM, dass Männer, } \\
\text { postmenopausale Frauen und } \\
\text { Schwangere Eisen nur nach } \\
\text { Rücksprache mit ihrem Arzt } \\
\text { einnehmen sollten }\end{array}$ \\
\hline Jod & $\mu \mathrm{g}$ & $150-200$ & $500\left[600^{*}\right]$ & $\begin{array}{l}149-175(\mathrm{~m})^{\star \star} \\
119-137(\mathrm{w})^{\star \star}\end{array}$ & $\begin{array}{l}246-377(m)^{\star \star} \\
195-239(\mathrm{w})^{\star \star}\end{array}$ & 100 & $\begin{array}{l}\text { NEM für schwangere und } \\
\text { stillende Frauen: } 150 \mu \mathrm{g} / \mathrm{Tag}\end{array}$ \\
\hline Fluorid & $\mathrm{mg}$ & $2,9-3,8$ & 7 & -- & -- & 0 & \\
\hline Zink & $\mathrm{mg}$ & $7,5-16,3^{\star \star \star}$ & 22 [25] & $\begin{array}{c}10,4-12,3(m) \\
8,6-9,5(w)\end{array}$ & $\begin{array}{l}16,8-22,9(m) \\
13,7-15,8(w)\end{array}$ & 6,5 & $\begin{array}{l}\text { Hinweis bei NEM mit mehr als } \\
3,5 \mathrm{mg} \text { Zink/Tag, auf die } \\
\text { Einnahme weiterer zinkhaltiger } \\
\text { NEM zu verzichten }\end{array}$ \\
\hline Mangan & $\mathrm{mg}$ & $2,0-5,0$ & -- & -- & -- & 0,5 & \\
\hline Silizium & $\mathrm{mg}$ & - & -- & 20 & -- & 50 & \\
\hline
\end{tabular}

a In Deutschland wurde der UL für Erwachsene, insbesondere zum Schutz älterer Personen, die lange einem Jodmangel ausgesetzt waren und dadurch möglicherweise ein erhöhtes Risiko für funktionelle Autonomien aufweisen, auf $500 \mu \mathrm{g} / \mathrm{Tag}$ festgesetzt

b Jodzufuhr unter der theoretischen Annahme, dass in 30 \% der verarbeiteten Lebensmittel Jodsalz verwendet wird (pers. Mitteilung v. T. Heuer, MRI)

c Zufuhrreferenzwerte der EFSA (2014a), die unter Berücksichtigung der in Europa beobachteten mittleren/medianen Phytataufnahmen $(300,600,900$ und $1200 \mathrm{mg} / \mathrm{Tag})$ abgeleitet wurden

signifikanten Beiträge zur Zufuhr leisten würden $(<10 \%$ des jeweiligen in der Verordnung (EU) $\mathrm{Nr}$. 1169/2011 angegebenen Referenzwertes).

Da bei Calcium insbesondere Frauen zwischen 14 und 18 Jahren die Zufuhrempfehlungen nicht erreichen, wird eine Höchstmenge von insgesamt $500 \mathrm{mg} /$ Tag vorgeschlagen, durch die ein signifikanter Beitrag zum Ausgleich von unzureichenden Aufnahmen über die Nahrung geleistet werden kann, ohne dass das Risiko für unerwünschte gesundheitliche Effekte in adäquat versorgten Bevölkerungsgruppen erheblich erhöht wird. Auch bei Zink wurden-auf Basis der Zufuhrempfehlungen der EFSA (2014a) $)^{2}$-Personen mit geringen

${ }^{2}$ Da die D-A-CH-Zufuhrreferenzwerte für Zink auf einer Ableitung aus dem Jahr 2000 basieren, wurde hier auf die aktuellen Werte der EFSA zurückgegriffen.
Zinkzufuhren (5. Zufuhrperzentile männlicher Personen) über die Nahrung berücksichtigt und eine Höchstmenge von $6,5 \mathrm{mg} /$ Tagesdosis eines NEM abgeleitet.

Bei den vorgeschlagenen Höchstmengen bleibt kein Spielraum für die Einnahme eines weiteren NEM mit Calcium oder Zink. Daher wird bei Zusätzen von mehr als $50 \%$ der jeweiligen Höchstmenge pro Tagesdosis eines NEM ein Hinweis empfohlen, auf den Verzehr weiterer NEM mit Calcium bzw. Zink zu verzichten.

\subsubsection{Magnesium}

Der für Magnesium abgeleitete UL von $250 \mathrm{mg} / \mathrm{Tag}$ betrifft ausschließlich zusätzliche Zufuhren (EFSA 2006). Magnesium aus der üblichen Ernährung kann daher bei der Höchstmengenableitung 
unberücksichtigt bleiben. Aufgrund der relativ leichten, vorübergehenden Effekte (milde Diarrhoe/ laxierende Effekte), auf denen die Ableitung des UL basiert, wird vorgeschlagen, den UL als Tageshöchstmenge für NEM zu übernehmen. Diese Option berücksichtigt keine Mehrfachexposition. Es wird jedoch empfohlen, die Tagesdosis von $250 \mathrm{mg}$ auf zwei oder mehr Zufuhreinheiten eines NEM zu verteilen.

\subsubsection{Eisen}

Die EFSA konnte auf der Basis der verfügbaren wissenschaftlichen Daten keine Dosis-Wirkungsbeziehung für die im Zusammenhang mit hohen Eisenaufnahmen diskutierten Krankheitsrisiken (kardiovaskuläre Krankheiten, Krebs und Diabetes mellitus Typ 2) und insgesamt keinen UL ableiten (EFSA 2006). Insbesondere gesunde erwachsene Männer, postmenopausale Frauen und Menschen mit hereditärer oder sekundärer Hämochromatose sind als Risikogruppen für negative gesundheitliche Wirkungen anzusehen. Diese Bevölkerungsgruppen sollten Eisen nur nach diagnostiziertem Mangel und unter ärztlicher Kontrolle supplementieren. Gleiches wird für Schwangere empfohlen, bei denen eine ärztliche Kontrolle des Eisenstatus in jedem Fall geboten ist. Da bei menstruierenden Frauen der Eisenbedarf erhöht ist und die Zufuhrreferenzwerte in den Altersgruppen der Frauen zwischen 14 und 50 Jahren im Median nicht erreicht werden, kannausschließlich für diese Altersgruppen-zur Höchstmengenableitung der UL des US-amerikanischen Institute of Medicine (IOM) (heute: National Academy of Medicine) von $45 \mathrm{mg} / \mathrm{Tag}$ (FNB 2002) herangezogen werden. Dem Ableitungsverfahren folgend, ergibt sich für Frauen zwischen ca. 14 und 50 Jahren eine Höchstmenge von $6 \mathrm{mg}$ pro Tagesdosis eines NEM. Es wird empfohlen, alle anderen Bevölkerungsgruppen durch einen entsprechenden Hinweis auf den Produkten vor einer unkontrollierten Eisensupplementierung zu warnen.

\subsubsection{Mangan}

Für Mangan konnte vom SCF wegen mangelnder Daten kein UL abgeleitet werden (SCF 2000 zit. in: EFSA 2006). Auch bestehen Unsicherheiten über die Manganaufnahme aus herkömmlichen Lebensmitteln. Für die Höchstmengenableitung wurde hilfsweise das Ergebnis einer EFSA-Bewertung herangezogen, die der britischen Expert Group on Vitamins and Minerals (EVM) gefolgt ist und festgestellt hat, dass zusätzliche Manganzufuhren von $4 \mathrm{mg} /$ Tag für die Allgemeinbevölkerung und von 0,5 mg/Tag für ältere Personen wahrscheinlich keine unerwünschten Wirkungen hervorrufen (EFSA 2009b). Da bei Erwachsenen unterschiedliche altersabhängige Dosierungen von Mangan in NEM nicht praktikabel erscheinen, wird empfohlen, die für ältere Personen als sicher angesehene Tagesdosis von $0,5 \mathrm{mg}$ als Höchstmenge für NEM festzusetzen. Unter diesen Bedingungen bleibt kein Spielraum für ein weiteres manganhaltiges NEM.

\subsubsection{Fluorid}

Da insbesondere aus fluoridhaltigem (Mineral-)Wasser und Zahnpflegemitteln bereits hohe Fluoridaufnahmen resultieren können (EFSA 2006) und in Deutschland fluoridiertes Speisesalz im Verkehr ist, sollte Fluorid nicht zusätzlich in NEM verwendet werden.

\subsubsection{Silizium}

Silizium ist kein anerkanntes essenzielles Spurenelement. Bisher wurde daher kein Zufuhrreferenzwert abgeleitet. Auch wurden in der NVS II keine Expositionsdaten erhoben. Ein UL konnte aufgrund fehlender Daten nicht abgeleitet werden (EFSA 2006). Über die normale Ernährung werden Siliziumaufnahmen zwischen 20 und $50 \mathrm{mg} / \mathrm{Tag}$ erreicht (EFSA 2006), die als sicher angesehen werden. Als Höchstmenge für NEM wird daher eine Menge vorgeschlagen, die in etwa der Zufuhr über die übliche Ernährung entspricht.

Die vorgeschlagenen Höchstmengen sind im Verhältnis zu den D-A-CH-Zufuhrreferenzwerten, den Aufnahmemengen aus der üblichen Nahrung (P50 und P95) und den jeweiligen ULs in den Tabs. 2 und 3 dargestellt.

\section{Diskussion und Schlussfolgerung}

In Deutschland werden durch die herkömmliche Ernährung bis auf wenige Ausnahmen ausreichende Mengen an Mikronährstoffen aufgenommen. Aus ernährungsphysiologischer Sicht sind NEM daher im Allgemeinen nicht notwendig. Dies gilt umso mehr, als NEM eher von Menschen mit gesünderem Lebensstil und ausgewogener Ernährung verwendet werden (Beitz et al. 2004; Rehaag et al. 2013; Rautiainen et al. 2014) und von einer zusätzlichen über den Bedarf hinausgehenden Aufnahme von 
Mikronährstoffen keine positiven Wirkungen zu erwarten sind (Bailey et al. 2011; Kang et al. 2016; Dharmarajan 2015). Angesichts dessen dienen die hier vorgeschlagenen Höchstmengen für den großen Teil der gut versorgten Bevölkerung zum Schutz vor übermäßigen Nährstoffaufnahmen, die langfristig mit einem erhöhten gesundheitlichen Risiko verbunden sein können. Sie basieren auf Expositionsschätzungen und Risikobewertungen hoher Nährstoffzufuhren unter Berücksichtigung der üblichen Ernährung und der (möglichen) Verwendung der Mikronährstoffe zur Lebensmittelanreicherung. Das im Jahr 2004 vom BfR erarbeitete Verfahren zur Ableitung von Höchstmengen für NEM und angereicherte Lebensmittel wurde in einem Vergleich mit anderen in Europa entwickelten Modellen zusammen mit dem von Rasmussen et al. (2006) als am besten geeignet eingestuft, um Verbraucher zu schützen (Dufour et al. 2010). Das BfR-Modell ist das einzige in Europa, das NEM und angereicherte Lebensmittel verknüpft. Dies ist wichtig, da Höchstmengen für eine der beiden Produktgruppen immer auch Auswirkungen auf die jeweils andere haben (Dufour et al. 2010; Lyhne Andersen und Tetens 2009).

Einschränkend muss festgestellt werden, dass das Modell nicht in jedem Fall anwendbar war, zum Beispiel dann nicht, wenn für den betreffenden Nährstoff vom SCF bzw. von der EFSA kein UL abgeleitet worden war oder für die deutsche Bevölkerung keine Nährstoffaufnahmedaten verfügbar sind. Bei einem Teil der Stoffe bestehen zudem erhebliche Wissenslücken und daher Unsicherheiten bei der Einschätzung des Gefährdungspotenzials. Insbesondere fehlen zum Teil adäquate Humandaten. Auch konnten Unterschiede in der individuellen Bioverfügbarkeit der Nährstoffe bzw. Stoffverbindungen und Interaktionen zwischen verschiedenen Nährstoffen oder mit anderen Lebensmittelinhaltsstoffen nicht oder nur ansatzweise berücksichtigt werden, zumal kaum systematische Informationen über die Bioverfügbarkeit der in NEM enthaltenen Vitaminund Mineralstoffverbindungen sowie über mögliche Interaktionen, auch mit Arzneimitteln, vorliegen (Yetley 2007). Einer der bekanntesten und am häufigsten beobachteten Interaktionen zwischen Vitamin $\mathrm{K}$ und gerinnungshemmenden Arzneimitteln vom Cumarintyp wurde jedoch durch die Empfehlung für einen Warnhinweis Rechnung getragen. Schließlich konnten keine (weiteren) Geschlechts- und Altersgruppenunterschiede sowie besondere physiologische Umstände oder spezielle Ernährungsmuster bei der Höchstmengenableitung berücksichtigt werden. Da NEM für die
Gesamtbevölkerung sicher sein sollen, wurden die Höchstmengen so abgeleitet, dass bei Personen ab 15 Jahren jeweils die Bedürfnisse der empfindlichsten Gruppe ausschlaggebend waren. Dafür wurden-soweit vorhanden-die niedrigeren ULs der 15- bis 17-Jährigen zugrunde gelegt, so dass die Produkte auch für diese Altersgruppe sicher sind.

Ferner wird-angesichts des weit verbreiteten Konsums von NEM in Deutschland (ca. $25 \%$ der NVS II-Population) und der Tatsache, dass ca. $25 \%$ der Supplementnehmer mehr als ein Produkt pro Tag verwenden (MRI 2008; Li et al. 2010; Heinemann et al. 2015)-ein Unsicherheitsfaktor von 2 zur Berücksichtigung möglicher Mehrfachexposition als gerechtfertigt angesehen, selbst wenn eine Mehrfachverwendung von NEM nicht zwangsläufig mit der wiederholten Aufnahme von gleichen Inhaltsstoffen einhergeht.

Abschließend sei darauf hingewiesen, dass die hier vorgeschlagenen Höchstmengen in Abhängigkeit von neuen wissenschaftlichen Erkenntnissen und zukünftigen Marktentwicklungen ggf. angepasst werden müssen. In jedem Fall wird ein begleitendes Monitoring empfohlen, um insbesondere bei Nährstoffen, für die keine Höchstmengen vorgeschlagen wurden, ggf. auf exzessive Dosierungen und dadurch bedingte hohe Nährstoffaufnahmen, die das Risiko für negative gesundheitliche Wirkungen erhöhen, reagieren zu können.

\section{Compliance with ethical standards}

Conflict of interest Die Autoren erklären, dass sie keine Interessenkonflikte in Zusammenhang mit diesem Artikel haben.

Open Access This article is distributed under the terms of the Creative Commons Attribution 4.0 International License (http://creativecommons.org/licenses/by/4.0/), which permits unrestricted use, distribution, and reproduction in any medium, provided you give appropriate credit to the original author(s) and the source, provide a link to the Creative Commons license, and indicate if changes were made.

\section{References}

Bailey RL, Fulgoni VL 3rd, Keast DR, Dwyer JT (2011) Dietary supplement use is associated with higher intakes of minerals from food sources. Am J Clin Nutr 94:1376-1381

Beitz R, Mensink GB, Hintzpeter B, Fischer B, Erbersdobler HF (2004) Do users of dietary supplements differ from nonusers in their food consumption? Eur J Epidemiol 19:335-341

BfR (2004a) Verwendung von Vitaminen in Lebensmitteln. Herausgegeben von Domke A, Großklaus R, Niemann B, 
Przyrembel H, Richter K, Schmidt E, Weißenborn A, Wörner B, Ziegenhagen R. BfR Wissenschaft 03/2004

BfR (2004b) Verwendung von Mineralstoffen in Lebensmitteln. Herausgegeben von Domke A, Großklaus R, Niemann B, Przyrembel H, Richter K, Schmidt E, Weißenborn A, Wörner B, Ziegenhagen R. BfR Wissenschaft 04/2004

BfR (2006). Höchstmengen für Bor und Fluorid in natürlichen Mineralwässern sollten sich an Trinkwasserregelungen orientieren. Stellungnahme Nr. 024/2006 des BfR vom 7. Februar 2006. http://www.bfr.bund.de/cm/343/ hoechstmengen_fuer_bor_und_fluorid_in_natuerlichen_ mineralwaessern_sollten_sich_an_ trinkwasserregelungen orientieren.pdf. Zugriff: 17.08.2017

Bruins MJ, Mugambi G, Verkaik-Kloosterman J, Hoekstra J, Kraemer K, Osendarp S, Melse-Boonstra A, Gallagher AM, Verhagen $H$ (2015) Addressing the risk of inadequate and excessive micronutrient intakes: traditional versus new approaches to setting adequate and safe micronutrient levels in foods. Food Nutr Res 59:26020

BVL/BfArM (2016) Stellungnahme der Gemeinsamen Expertenkommission BVL/BfArM. Bewertung von Vitamin-Dhaltigen Produkten (01/2016). https://www.bvl.bund.de/ SharedDocs/Downloads/01_Lebensmittel/ expertenkommission/Zweite_Stellungnahme_VitaminD. pdf?_blob=publicationFile\&v=8. Zugriff: 07.03.2017

Deutsche Gesellschaft für Ernährung, Österreichische Gesellschaft für Ernährung, Schweizerische Gesellschaft für Ernährung (2015) Referenzwerte für die Nährstoffzufuhr. 2. Auflage, 1. Ausgabe 2015, Neuer Umschau Buchverlag

DGE (2012) 12. Ernährungsbericht 2012. Deutsche Gesellschaft für Ernährung e. V. (Hrsg.), Bonn

Dharmarajan TS (2015) Is vitamin supplementation appropriate in the healthy old? Curr Opin Gastroenterol 31:143-152

Dufour A, Wetzler S, Touvier M, Lioret S, Gioda J, Lafay L, Dubuisson C, Calamassi-Tran G, Kalonji E, Margaritis I, Volatier JL (2010) Comparison of different maximum safe levels in fortified foods and supplements using a probabilistic risk assessment approach. Br J Nutr 104:1848-1857

EFSA European Food Safety Authority (2006) Tolerable upper intake levels for vitamins and minerals. Scientific committee on food, Scientific panel on dietetic products, Nutrition and allergies. EFSA. http://www.efsa.europa.eu/ sites/default/files/efsa_rep/blobserver_assets/ ndatolerableuil.pdf. Zugriff: 31.10.2017

EFSA European Food Safety Authority (2009a) Scientific opinion of the panel on food additives and nutrient sources added to food on inositol hexanicotinate (inositol hexaniacinate) as a source for niacin (vitamin B3) added for nutritional purposes in food supplements following a request from the European Commission. EFSA J 949:1-20

EFSA European Food Safety Authority (2009b) Scientific opinion of the panel on food additives and nutrient sources added to food on manganese ascorbate, manganese aspartate, manganese bisglycinate and manganese pidolate as sources of manganese added for nutritional purposes to food supplements following a request from the European Commission. EFSA J 1114:1-1123

EFSA European Food Safety Authority (2012) Statement on the safety of $\beta$-carotene use in heavy smokers. EFSA J 10:2953

EFSA European Food Safety Authority (2013) Scientific opinion on dietary reference values for molybdenum. EFSA J 11:3333

EFSA European Food Safety Authority (2014a) Scientific opinion on dietary reference values for zinc. EFSA J 12:3844
EFSA European Food Safety Authority (2014b) Scientific opinion on dietary reference values for selenium. EFSA J 12:3846

EFSA European Food Safety Authority (2015) Scientific opinion on dietary reference values for copper. EFSA J 13:4253

Flynn A, Moreiras O, Stehle P, Fletcher RJ, Müller DJ, Rolland V (2003) Vitamins and minerals: a model for safe addition to foods. Eur J Nutr 42:118-130

FNB Food and Nutrition Board (2001) Dietary reference intakes: applications in dietary assessment. Institute of Medicine, National Academy Press, Washington

FNB Food and Nutrition Board (2002) Dietary reference intakes for vitamin A, vitamin $\mathrm{K}$, arsenic, boron, chromium, copper, iodine, iron, manganese, molybdenum, nickel, silicon, vanadium, and zinc. Institute of Medicine, National Academic Press, Washington, pp 290-393

Gerber N (2014) Risikobewertung der Aufnahme von isoliertem Beta-Carotin aus angereicherten Lebensmitteln sowie Nahrungsergänzungsmitteln im Hinblick auf die Festlegung eines gesetzlichen Höchstwertes für den Zusatz als Provitamin zu Lebensmitteln. Masterarbeit im Studiengang Master of advanced Studies in Food Safety Management, Universität Basel (Advanced Study Centre), Basel. https://www.openagrar.de/receive/openagrar_ mods_00011050. Zugriff: 31.10.2017

Heinemann M, Willers J, Bitterlich N, Hahn A (2015) Verwendung von Nahrungsergänzungsmitteln mit Vitaminen und Mineralstoffen-Ergebnisse einer deutschlandweiten Verbraucherbefragung. J Verbr Lebensm 10:131-142

Kang M, Kim DW, Lee H, Lee YJ, Jung HJ, Paik HY, Song YJ (2016) The nutrition contribution of dietary supplements on total nutrient intake in children and adolescents. Eur J Clin Nutr 70:257-261

Kloosterman J, Fransen HP, de Stoppelaar J, Verhagen $\mathrm{H}$, Rompelberg C (2007) Safe addition of vitamins and minerals to foods: setting maximum levels for fortification in the Netherlands. Eur J Nutr 46:220-229

Li K, Kaaks R, Linseisen J, Rohrmann S (2010) Consistency of vitamin and/or mineral supplement use and demographic, lifestyle and health-status predictors: findings from the European Prospective Investigation into Cancer and Nutrition (EPIC)-Heidelberg cohort. $\mathrm{Br} \mathrm{J}$ Nutr 104:1058-1064

Lyhne Andersen N, Tetens I (2009) How to reach a common estimate of high dietary micronutrient intakes for safe addition of vitamins and minerals to foods? Food Nutr Res 53:1898. https://doi.org/10.3402/fnr.v53i0.1898

Mensink GBM, Heseker H, Richter A, Stahl A, Vohmann C (2007) Ernährungsstudie als KiGGS-Modul (EsKiMo). Robert Koch-Institut, Universität Paderborn

Miller ER, Pastor-Barriuso R, Dalal D, Riemersma RA, Appel LJ, Guallar E (2005) Meta-analysis: high-dosage vitamin E supplementation may increase all-cause mortality. Ann Intern Med 142:37-46

MRI (2008) Max Rubner-Institut. Nationale Verzehrstudie II, Ergebnisbericht, Teil 2. Max Rubner-Institut, Bundesforschungsinstitut für Ernährung und Lebensmittel

NemV Nahrungsergänzungsmittelverordnung vom 24. Mai 2004 (BGBl. I S. 1011), die zuletzt durch Artikel 11 der Verordnung vom 5. Juli 2017 (BGBl. I S. 2272) geändert worden ist. https://www.gesetze-im-internet.de/nemv/ NemV.pdf Zugriff: 31.10.2017

Rasmussen SE, Andersen NL, Dragsted LO, Larsen IC (2006) A safe strategy for addition of vitamins and minerals to foods. Eur J Nutr 45:123-135 
Rautiainen S, Wang L, Gaziano JM, Sesso HD (2014) Who uses multivitamins? A cross-sectional study in the Physicians' Health Study. Eur J Nutr 53:1065-1072

Rehaag R, Tils G, Röder B, Ulbig E, Kurzenhäuser-Carstens S, Lohmann M, Böl G-F (2013) Zielgruppengerechte Risikokommunikation zum Thema Nahrungsergänzungsmittel. Abschlussbericht. Bundesinstitut für Risikobewertung (Hrsg), Berlin

Schürks M, Glynn RJ, Rist PM, Tzourio C, Kurth T (2010) Effects of vitamin $\mathrm{E}$ on stroke subtypes: meta-analysis of randomised controlled trials. BMJ 341:c5702
Verkaik-Kloosterman J, McCann MT, Hoekstra J, Verhagen H (2012) Vitamins and minerals: issues associated with too low and too high population intakes. Food Nutr Res 56:5728. https://doi.org/10.3402/fnr.v56i0.5728. https://www.ncbi.nlm. nih.gov/pmc/articles/PMC3321245/pdf/FNR-56-5728.pdf

Yetley EA (2007) Multivitamin and multimineral dietary supplements: definitions, characterization, bioavailability, and drug interactions. Am J Clin Nutr 85:269S-276S 